\title{
A Conceptual Approach to Enhance the Well-Being of Elderly People
}

\author{
Diogo Martinho ${ }^{1[0000-0003-1683-4950]}$, João Carneiro ${ }^{1[0000-0003-1430-5465]}$, Paulo Novais ${ }^{2[0000-}$ \\ 0002-3549-0754], José Neves ${ }^{2[0000-0002-8863-0351]}$, Juan Corchado ${ }^{3[0000-0002-2829-1829]}$, Goreti \\ Marreiros 1 [0000-0003-4417-8401] \\ ${ }^{1}$ Research Group on Intelligent Engineering and Computing for Advanced Innovation and \\ Development (GECAD), Institute of Engineering, Polytechnic of Porto, Porto, Portugal \\ diepm, jomrc, mgt @isep.ipp.pt \\ ${ }^{2}$ ALGORITMI Centre, University of Minho, Guimarães, Portugal \\ pjon, jneves @di.uminho.pt \\ ${ }^{3}$ BISITE Digital Innovation Hub, University of Salamanca. Edificio Multiusos \\ corchadodusal.es
}

\begin{abstract}
The number of elderly people living alone is increasing. Consequently, a lot of research works have been addressing this issue in order to propose solutions that can enhance the quality of life of elderly people. Most of them have been concerned in dealing with objective issues such as forgetfulness or detecting falls. In this paper, we propose a conceptual approach of a system that intends to enhance the daily sense of user's well-being. For that, our proposal consists in a system that works as a social network and a smartwatch application that works unobtrusively and collects the user's physiological data. In addition, we debate how important features such as to detect user's affective states and to potentiate user's memory could be implemented. Our study shows that there are still some important limitations which affect the success of applications built in the context of elderly care and which are mostly related with accuracy and usability of this kind of system. However, we believe that with our approach we will be able to address some of those limitations and define a system that can enhance the wellbeing of elderly people and improve their cognitive capabilities.
\end{abstract}

Keywords: Ambient Assisted Living, Cognitive Assistant, Affective Wearables, Affective Computing, Gamification, Elderly Care.

\section{Introduction}

We are currently witnessing an increase of the elderly population around the world. According to the United Nations's World Population Ageing Report [1], the global population aged 60 years or older has registered a total of 962 million in 2017 and it is expected to reach 2 billion by 2050. With such an increase of the number of older citizens living in today's society it becomes necessary to research and develop assistive technologies that can support elderly people $[2,3]$ and encourage them to maintain independence [4]. It no longer makes sense to just rely on traditional healthcare services 
to assist elderly people (such as adult day cares, or nursing homes) which are expensive and unfordable by many, and that cannot accurately assess the current health condition of the elderly (both physical and cognitive). Therefore, physical environments enhanced by innovative technologies should become the new trend in the context of elderly care to promote active ageing. These physical environments also known as smart homes [4-6] comprise a set of technologies including telecare devices [7], persuasive technologies [8], rehabilitation systems [9] and digital games [10-13].

In this work, we propose a conceptual approach to enhance the well-being of elderly people. Our approach differs from most existent approaches because it concerns in potentiating the elders' sense of well-being through activities that control their levels of stress, anxiety and cognitive capabilities, rather than focusing in most traditional tasks such as detecting falls, scheduling meds, among others. We propose (conceptually) a social network specific for elderly people, where they can interact naturally with their family members and other elders and a smartwatch application that controls the physiological signals and work together with the system (social network) to study the effect of different interactions on the person. Furthermore, an overview of the current state of the literature regarding different topics relevant to the context of this work is also presented so that we can understand existing knowledge that allows us to develop the proposed approach and also allows us to understand what kind of main limitations and challenges should be overcome.

The rest of the paper is organized in the following order: in the next Section we present the related work. In Section 3 we described the proposed conceptual approach, mostly in terms of architecture, features and devices. Finally, some conclusions are put forward in Section 4, alongside with suggestions of work to be done hereafter.

\section{$2 \quad$ Related Work}

In this work we explored some of the most recent approaches under the topics of cognitive assistants, affective wearables and gamification applied to healthcare with focus on elderly care. We believe these three areas of artificial intelligence are essential to define a personalized approach to correctly support elderly people and motivate them to pursuit healthier lifestyle habits. For this, cognitive assistants can provide tools and features to help people perform activities of daily living [14]. Furthermore, these assistants will interact directly with the user using different mechanisms such as affective wearables to collect physiological data and correctly assess user's affective states and gamification to motivate the user to perform different activities and improve health condition.

\subsection{Cognitive Assistants}

The development and research of solutions using cognitive assistants has seen great advances in the literature over the last decade. Cognitive assistants can be endowed with social and emotional processing $[15,16]$ to interact with humans and improve their capabilities while not replacing them in specific tasks. In other words, cognitive 
assistants will augment human intelligence and assist in decision-making and action taking [17]. As such, in the last years there have been proposed very interesting approaches targeted towards elderly care with the goal to enhance their daily lives and improve both their cognitive and physical capabilities. Costa et al. [18] proposed an interactive physical robot system to recommend and monitor physical exercises designed for the elderly people. The system is divided in three main components: a physical robot which interacts with the elderly person and records the performance of different exercises.; a human exercise recognition system which uses deep learning techniques to identify different gestures associated to each exercise; and a recommendation system which suggests and adapts physical activities according to the capabilities and current health condition of the elderly person. The authors were able to validate the developed system in terms of exercise variance and personalization; however, they still recognized some limitations mainly related with the accuracy of their exercise recognition algorithm and the lack or robot gestures mimicking the exerciser. Kostavelis et al. [19] proposed a service robot system to operate in domestic environments and support elderly people and people with mild cognitive impairments. For this, the developed robot included a set of mechanisms to observe and perceive the environment, track different activities and assess cognitive and physical capabilities. The authors presented the architecture of the developed system under both hardware and software point of view and highlighted how the developed system should discreetly assist the user in daily activities and allow to retain both autonomy and decisionmaking. Furthermore, the authors also concluded that the developed system should never replace the caregiver but act as a complementary to the work of the caregivers instead. Ramos et al. [14] proposed a system using a smartphone and augmented reality to guide people with mild or moderate cognitive disabilities according to their preferences and offers an application that allows the caregiver to locate the disabled person. The developed system also considered ethical questions since it collects and ciphers sensitive data and assumes the disabled person has given his/her consent to provide all the information necessary for the system to operate correctly. The authors were not able to test and validate the proposed system in real case scenarios and they expressed it would be necessary to select people with cognitive impairments for further system testing and evaluation as the only tests performed in this study used people without any cognitive disabilities. Nguyen-Thinh and Laura [20] proposed a cognitive assistant to improve the reasoning and decision-making ability of users by teaching the user different topics while holding a conversation with them using natural language processing and a dialog model. The authors performed a study to validate the developed model with 65 participants and obtained positive feedback regarding the interaction with the system, however the authors expressed a larger sample as well as a larger testing timespan would be necessary to further validate the results of the presented study. The robot developed in [21] promotes social interaction between elderly people while performing different functions such as: monitoring and recognizing facial expressions which translate to different emotions and adapting the interaction with each elderly person; playing different games with elderly people so they keep interested to interact with the robot; personalizing care with flexible communication features like speech recognition and voice vocalization; promoting mental activity while running a 
set of short or long quizzes which depend on the alertness and medical condition of the elderly person. A field trial was performed with seventy residents of residential homes over a three-day period and the authors obtained positive results regarding engagement, acceptability, personalization and facilitation of healthier eating and living. In [22], three robots were developed for a preventive care system which are able to interact and compete against each other during a match up game based on a traditional Japanese game. The system adapts the exercise load according to the feedback provided by the user (the user reports the fatigue level in a questionnaire presented in a tablet device). The authors performed three experiments to test their system and the result showed that elderly people preferred to play the game rather than watch others playing it. However, the developed system only provided accurate exercise loads for $70 \%$ of the participants. Furthermore, the authors also expressed it would be relevant to extend the game settings and test the developed system with multiple players at the same time.

\subsection{Affective Wearables: Detection of emotions, stress and anxiety}

The affective wearables consist in systems composed by sensors and tools to recognize its wearer's affective patterns such as friendly gesture, a strange voice, expression of emotions or a change in autonomic nervous system activity (such as heart rate or skin conductivity) [23]. The data collected by affective wearables can then be used to help wearers, improving their quality of life or prevent the occurrence of negative events. Since affective wearables are usually in contact with the user during a considerable time period and may take the form of clothes, jewelry, watches, among others [24, 25], these have less constraints than other traditional devices such a keyboard or a mouse. This ability to recognize physical and psychological patterns allows to develop intelligent systems capable of adapting to the needs of each user [26-29]. One of the current greatest challenges in the development of affective wearables consists in turning a system capable of recognizing different affective patterns. If on the one hand the affective wearables allow to collect important data, on the other hand it is hard to create "rules" to determine which responses occur with which emotions, as different individuals can feel different emotions and demonstrate different behaviors in the same situation. Several authors have been addressed this problematic in the recent years. Yang and Samuel [30] proposed a method to detect user's emotions based on the user's voice. Their approach consists in detecting the user's voice using the smartphone's microphone and consequently use a liner machine learning algorithm to train the system and to predict the emotions. They method allows to predict happy and sad emotions with an accuracy of $71 \%$ Dai, Liu and Meng [31] proposed an approach that intends to understand the user's emotional state according to the usage of smartphone. They used the finger-stroke pattern to predict the user's emotional state (positive, neutral, and negative). A machine learning classification algorithm to train the classifier was used and the results showed an accuracy of 72,3\%,74,6\% and 69\% for male, female and all subjects. Hänsel, Alomainy and Haddadi [32] presented a smartphone/smartwatch application that allows users to indicate the affective state they are feeling. They use the data collected from the smartphone and smartwatch such as current location, heart rate, prior physical activity (steps and workouts), ambient noise and wrist movements 
to correlate with the reported affective states. No results were reported but the authors intend to evaluate the application and to study if the collected data can be used to infer emotional and affective states. Ciabattoni, Ferracuti, Longhi, Pepa, Romeo and Verdini [33] proposed an approach to detect mental stress in real-time. They used a smartwatch to collect bio signal data (heart rate, galvanic skin response and body temperature). They chose a smartwatch because they intended to detect the stress through an unobtrusively and inexpensive way. The Mutual Information (M.I.) was used to measure the dependence between variables and the features with the highest M.I. were selected to train a K-NN classifier. The results obtained indicated an accuracy of 84,5\% and a misclassification error of $26 \%$ when subject is relaxed. There are also some other works where the authors use similar strategies and that present similar results, such as the one proposed by Quiroz, Geangu and Yong [34] (median accuracies higher than 78\%) and the one proposed by Zhang, Song, Cui, Liu and Zhu [35] (with an accuracy that ranged from $60 \%$ to $91,3 \%$ ).

\subsection{Gamification}

The use of games and game design elements in nongame contexts is being applied to different areas and application fields such as healthcare, digital marketing, finances, education, productivity, sustainability or even news [36, 37]. Two concepts have emerged in the literature also known as serious games and gamification. Serious games can be described as games "designed to entertain players as they educate, train, or change behavior" [38], while gamification refers to the enhancement of services with features that can offer "gameful" experiences to its and motivate and engage them in the pursuit of different activities, social interaction, and to increase the quality and productivity of their actions [39]. Recent research and advancements on gamification and serious games have been made in healthcare, and more particularly, in elderly care. Gamification and serious games are now being study as a way to motivate and persuade elderly people to undertake both physical, cognitive and social activities, thus contributing to their overall wellbeing and to motivate elderly people to pursuit more active and healthy lifestyles [11, 40, 41]. In [42] it is presented a smartwatch application to monitor and support people suffering from obesity and to motivate them to follow diet programs. The authors refer to the fact that our current lifestyle habits such as sedentary behaviors and poor diets contribute to chronic illness and to experience longterm limitations such as obesity until death. The authors claim that these issues cannot be simply managed through a set of measurements on patient health state nor through a set of treatments, but instead require day-to-day planning and adherence to new and healthier behaviors for best health outcomes in a long-term. They explain how personalized diet programs should be presented to the patient using checklists providing specific set of activities and performance goals that are embedded in daily life activities. The authors finally express that their approach will allow to document patient past successful experiences and increase their self-efficacy and contribute to healthier lifestyle changes and long-term health outcomes. In [43] it is performed a study to evaluate the efficacy of a smartwatch intervention in home-based dementia care. The authors explain how current home-based care models that monitor patients and report 
the progression of their physical and cognitive degeneration depend on health operators visits and these visits are scheduled based on passage of time and not based on the cognitive and physical current condition of the patient [44].To overcome this issue, the authors then explain how smartwatches could be used to monitor physical health aspects of dementia patients in a home-based care scenario and provide physicians with relevant information regarding events or activities experienced by the patient which cannot be recollected or re-experienced during a home visit. In [25], the authors discuss how gamification elements may improve user behavior and health condition using wearable self-tracking devices (such as smartphones or smartwatches). However, the authors explain that it is currently not know how users of these kind of devices accurately perceive their motivations being fulfilled nor how the use of gamification can raise motivation and device usage. To study these issues the authors developed a conceptual model where they investigated the influence of motivational factors by combining two research models developed by Gimpel et al. [45] and by Baumgart and Wiewiorra [24] with the integration of gamification elements and explained the many different advantages of using gamification elements in this kind of devices (to enhance user experience, ensure user engagement and to contribute to long-term adherence). The authors still alert to some of the negative aspects of using gamification elements in self-tracking devices (elements such as rewards and social comparison might contribute to decreasing autonomy and intrinsic motivation). Finally, the authors conclude that the usage of gamification elements should not be mandatory and should be up to each user instead.

\section{Proposed (Conceptual) Approach}

This work distinguished essentially by the way how the problematic of elderly care is addressed. Rather than idealize the system in terms of a model capable of detecting falls or scheduling meds, our focus is to increase the sense of well-being in cognitive and affective terms. Obviously, this topic is neither more nor less important than others, but it is a less traditional topic in this context.

This approach was planned with three main concerns in mind: scalability, unobtrusively and affordable. The cheapest way to use the system is using an internet connection, a smartwatch and a desktop/laptop/smartphone/tablet. So, this baseline version consists in an internet connection and two devices. The smartwatch works in the user's perspective as any regular watch in order to respect our concern in maintain the system unobtrusive. The system's smartwatch application collects physiological data of the elderly person (such as heart rate, body temperature, etc.) through embedded sensors, and sends this information plus other relevant data (such as the date and time when a certain physiological data was collected) to an online server to be processed. After this, the system may send a response (if necessary) to the user through the tablet application to recommend different cognitive activities (these activities are detailed in the Features section of this work). The most expensive way to use the system would be through several sensors and tablets installed through the elder person's home and detecting and collecting additional data (user location, fall detection, user actions such 
as cooking or sleeping or bathing) and providing more personalized interactions (for example, having a tablet installed in the bathroom and using a visual reminder with a photo of a family member to remind the elderly person to brush teeth and take medication).

\subsection{Architecture}

In this section we present the architecture for the system proposed in this work.

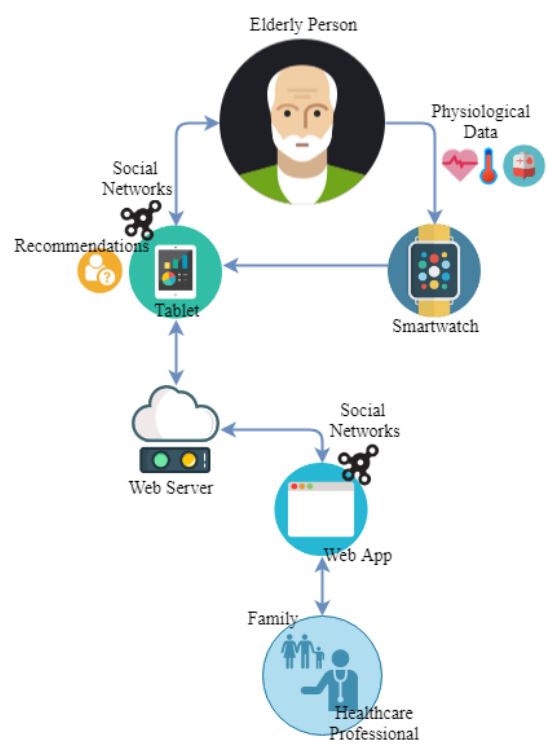

Fig. 1. Proposed System Architecture

As can be seen in Fig. 1, the main user of the system is the elderly person which interacts directly with the smartwatch and tablet. Regarding the interaction with the smartwatch, the relation is unidirectional as the elderly person will provide physiological data which is captured by the smartwatch embedded sensors. With this flow we can assure an unobtrusive collection of data without user explicit interference. All the information acquired by the smartwatch along with other relevant information that can be measured and acquired using the smartwatch is sent to the table device. The tablet receives all the acquired data from the smartwatch as well as any kind of user direct input while using the application. This data is then sent to the web server to be processed in order to generate specific recommendations/suggestions regarding different activities that can be performed by the elderly person. The application also includes a web component that can be accessed remotely by family members or healthcare professionals to check and assess the current health condition of the elderly person as well as to exchange messages, images, videos, or even recommendations and suggestions on more activities to be performed by the elderly person. We consider that the proposed system will offer a set of intelligent features that will allow to establish a 
more intelligent and personalized interaction with the elderly person based on his health condition, capabilities, preferences, interests and affective state. Technologies considered for the proposed architecture include but are not limited to: (1) the use of Xamarin ${ }^{1}$ which allows smartwatch app development; (2) the use of Drools Business Rule Management System ${ }^{2}$ as inference based rules engine which allows for simplified understanding of coding, flexibility, reusability, centralized knowledge, scalability among other benefits. In terms of devices, the only requirement considered is the capability of collecting physiological data and there already several solutions available in the market such as Xiaomi Smart Band ${ }^{3}$ and Samsung Galaxy Fit ${ }^{4}$ as more affordable options and Sony SmartWatch ${ }^{5}$ and Apple Watch ${ }^{6}$ as more expensive options.

\subsection{Features}

In our approach we present three main features which represent the novelty of work and which allow to develop a fully personalized system that can correctly interact with the elderly person and motivate to be healthier and at the same time improve cognitive capabilities. These features are as follows:

To learn user's preferences and potentiate his/her fun. Besides including different features available in the most known social networks available online (such as exchanging messages, comments, likes and dislikes, among others), we propose a social network adapted to the context of elderly care whose information provided to the user must follow his preferences, interests and necessities. Moreover, it must understand the impact associated with each provided information as to understand if the user perceived that information as a positive or negative event. For this, in our approach we consider different physiological data that will be acquired while the elderly person is interacting with the social network and then processed using big data and different machine learning algorithms. After that, we can define the profile of the elderly person and identify and predict possible interests and preferences and this way personalize the social network accordingly. As a result, the elderly person will feel interested and motivated to use the social network, and ultimately be entertained while doing so.

To evaluate the current user's cognitive capabilities and to work on strategies that can help him/her stimulate his/her memory. With this feature, we intend to evaluate and measure the cognitive state of the elderly person as well as the cognitive degradation overtime. For this, we consider the use of gamification techniques to assess the memory of the elderly person such as to suggest effective recommendations and activities to stimulate his/her cognitive capabilities and to understand if the elderly

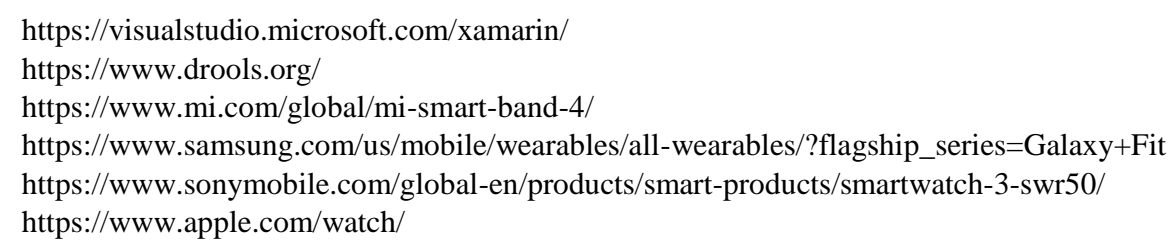


person had improved or worsen his/her ability to perform the same activity overtime. These activities are presented to the elderly person using different game structures (such as quizzes, puzzles, associations, etc.) and the elderly person will then be able to build own family tree and recall past events and experiences. Furthermore, we combine both these gamification techniques with the proposed social network (and the retrieved data) to promote social interaction between the elderly person and his/her family members (for example, using family photos and videos). This way we can motivate the elderly person to use the system, and to define intelligent strategies that can improve his/her memory and cognitive condition due to all factors that involve and that may affect the family context of the elderly person. Besides this, this feature considers the two current major limitations associated to gamified systems related with the lack of personalization of the support provided to each individual (as the system only provides the same features to every single user instead) and with the fact that elderly people have difficulty to understand, accept and use new technologies and game mechanics that are available in gamified approaches (resulting in a great difficulty to perceive the inherent benefits associated to those systems). Therefore, all suggested activities and gamification techniques used to measure and assess cognitive condition must be presented to each user according to his/her interests and preferences.

To detect affective states, stress and anxiety. The user affective state, stress and anxiety levels are monitored through the smartwatch application to process physiological data with each interaction to understand what kind of events and interactions are perceived as positive or negative by the elderly person. For this, big data analysis is performed, and the acquired data is compared with pre-established patterns (for example, ECG patterns) for different daily activities performed by the elderly person. By doing so, the system will be capable of personalizing and filter negative interactions with the user. According to the literature, it is still very difficult to develop a system that can accurately measure and identify generated emotions using this kind of approach

\section{Conclusions and Future Work}

In this paper we proposed a conceptual approach that intends to increase the sense of well-being of elderly people. In this regard, we presented a conceptual version of a system that works as a social network and that can be used in any common device such as a smartphone, a tablet, a laptop or a desktop. In addition, the system includes a smartwatch application which gathers user's physiological signs and sends them to the server. Considering the environment that a social network can provide and the acquired data, we proposed strategies to achieve three major features: (1) to learn user's preferences and potentiate his/her fun; (2) to evaluate the current user's cognitive capabilities and to work on strategies that can help stimulate memory (refreshing his/her memory associations); and (3) to detect affective states, stress and anxiety.

We concluded that the biggest challenges are to develop gamification strategies that can be adequate to the different needs and capabilities of each user, to develop strategies 
that can accurately detect user's emotions and to develop a model that can learn the user's preferences, maintaining at same time a level of usability adequate to the user's profile.

As future work, we want to study strategies that allow to overcome the limitations that were identified in each presented feature. We also intend to develop a prototype to evaluate the models and algorithms that will be created and finally we intend to test the prototype in a real scenario.

\section{Acknowledgments}

The work presented in this paper has been developed under the EUREKA - ITEA3 Project PHE (PHE-16040), and by National Funds through FCT (Fundação para a Ciência e a Tecnologia) under the projects UID/EEA/00760/2019 and UID/CEC/00319/2019 and by NORTE-01-0247-FEDER-033275 (AIRDOC “Aplicação móvel Inteligente para suporte individualizado e monitorização da função e sons Respiratórios de Doentes Obstrutivos Crónicos") by NORTE 2020 (Programa Operacional Regional do Norte).

\section{References}

1. UN: World Population Ageing Report. (2017)

2. Durick, J., Robertson, T., Brereton, M., Vetere, F., Nansen, B.: Dispelling ageing myths in technology design. In: Proceedings of the 25th Australian Computer-Human Interaction Conference: Augmentation, Application, Innovation, Collaboration, pp. 467-476. ACM, (2013) 3. Roupa, Z., Nikas, M., Gerasimou, E., Zafeiri, V., Giasyrani, L., Kazitori, E., Sotiropoulou, P.: The use of technology by the elderly. Health Science Journal 4, 118 (2010)

4. Do, H.M., Pham, M., Sheng, W., Yang, D., Liu, M.: RiSH: A robot-integrated smart home for elderly care. Robotics and Autonomous Systems 101, 74-92 (2018)

5. Cook, D., Das, S.K.: Smart environments: Technology, protocols and applications. John Wiley \& Sons (2004)

6. Liu, L., Stroulia, E., Nikolaidis, I., Miguel-Cruz, A., Rincon, A.R.: Smart homes and home health monitoring technologies for older adults: A systematic review. International journal of medical informatics 91, 44-59 (2016)

7. Botsis, T., Demiris, G., Pedersen, S., Hartvigsen, G.: Home telecare technologies for the elderly. Journal of Telemedicine and Telecare 14, 333-337 (2008)

8. Chatterjee, S., Price, A.: Healthy living with persuasive technologies: framework, issues, and challenges. Journal of the American Medical Informatics Association 16, 171-178 (2009)

9. Organization, W.H.: Rehabilitation in health systems. (2017)

10.Van Diest, M., Lamoth, C.J., Stegenga, J., Verkerke, G.J., Postema, K.: Exergaming for balance training of elderly: state of the art and future developments. Journal of neuroengineering and rehabilitation 10, 101 (2013)

11.Gerling, K.M., Masuch, M.: Exploring the potential of gamification among frail elderly persons. In: Proceedings of the CHI 2011 Workshop Gamification: Using Game Design Elements in Non-Game Contexts. (2011) 
12.Gerling, K.M., Schulte, F.P., Masuch, M.: Designing and evaluating digital games for frail elderly persons. In: Proceedings of the 8th international conference on advances in computer entertainment technology, pp. 62. ACM, (2011)

13.Ijsselsteijn, W., Nap, H.H., de Kort, Y., Poels, K.: Digital game design for elderly users. In: Proceedings of the 2007 conference on Future Play, pp. 17-22. ACM, (2007)

14.Ramos, J., Oliveira, T., Satoh, K., Neves, J., Novais, P.: Cognitive assistants-An analysis and future trends based on speculative default reasoning. Applied Sciences 8, 742 (2018)

15.Paiva, A., Mascarenhas, S., Petisca, S., Correia, F., Alves-Oliveira, P.: Towards more humane machines: Creating emotional social robots. New Interdisciplinary Landscapes in Morality and Emotion, pp. 125-139. Routledge (2018)

16.Leite, I., Martinho, C., Paiva, A.: Social robots for long-term interaction: a survey. International Journal of Social Robotics 5, 291-308 (2013)

17.Engelbart, D.C.: Augmenting human intellect: A conceptual framework. Menlo Park, CA (1962)

18.Costa, A., Martinez-Martin, E., Cazorla, M., Julian, V.: PHAROS—PHysical assistant RObot system. Sensors 18, 2633 (2018)

19.Kostavelis, I., Giakoumis, D., Malasiotis, S., Tzovaras, D.: Ramcip: Towards a robotic assistant to support elderly with mild cognitive impairments at home. In: International Symposium on Pervasive Computing Paradigms for Mental Health, pp. 186-195. Springer, (2015)

20.Le, N.-T., Wartschinski, L.: A Cognitive Assistant for improving human reasoning skills. International Journal of Human-Computer Studies 117, 45-54 (2018)

21.Khosla, R., Chu, M.-T., Nguyen, K.: Affective Robot Enabled Capacity and Quality Improvement of Nursing Home Aged Care Services in Australia. In: Computer Software and Applications Conference Workshops (COMPSACW), 2013 IEEE 37th Annual, pp. 409-414. IEEE, (2013)

22.Kitakoshi, D., Okano, T., Suzuki, M.: An empirical study on evaluating basic characteristics and adaptability to users of a preventive care system with learning communication robots. Soft Computing 21, 331-351 (2017)

23.Picard, R.W., Healey, J.: Affective wearables. Personal Technologies 1, 231-240 (1997)

24.Baumgart, R., Wiewiorra, L.: The Role of Self-Control in Self-Tracking. (2016)

25.von Entress-Fürsteneck, M., Gimpel, H., Nüske, N., Rückel, T., Urbach, N.: Self-Tracking and Gamification: Analyzing the Interplay of Motivations, Usage and Motivation Fulfillment. (2019)

26.Kostopoulos, P., Kyritsis, A.I., Ricard, V., Deriaz, M., Konstantas, D.: Enhance daily live and health of elderly people. Procedia computer science 130, 967-972 (2018)

27.O'Connor, D., Brennan, L., Caulfield, B.: The use of neuromuscular electrical stimulation (NMES) for managing the complications of ageing related to reduced exercise participation. Maturitas 113, 13-20 (2018)

28.Malwade, S., Abdul, S.S., Uddin, M., Nursetyo, A.A., Fernandez-Luque, L., Zhu, X.K., Cilliers, L., Wong, C.-P., Bamidis, P., Li, Y.-C.J.: Mobile and wearable technologies in healthcare for the ageing population. Computer methods and programs in biomedicine 161, 233237 (2018) 
29.Gamecho, B., Silva, H., Guerreiro, J., Gardeazabal, L., Abascal, J.: A context-aware application to increase elderly users compliance with physical rehabilitation exercises at home via animatronic biofeedback. Journal of medical systems 39, 135 (2015)

30.Yang, N., Samuel, A.: Context-rich detection of user's emotions using a smartphone. Microsoft Research Internship Report (2011)

31.Dai, D., Liu, Q., Meng, H.: Can your smartphone detect your emotion? In: 2016 12th International Conference on Natural Computation, Fuzzy Systems and Knowledge Discovery (ICNC-FSKD), pp. 1704-1709. IEEE, (2016)

32.Hänsel, K., Alomainy, A., Haddadi, H.: Large scale mood and stress self-assessments on a smartwatch. In: Proceedings of the 2016 ACM International Joint Conference on Pervasive and Ubiquitous Computing: Adjunct, pp. 1180-1184. ACM, (2016)

33.Ciabattoni, L., Ferracuti, F., Longhi, S., Pepa, L., Romeo, L., Verdini, F.: Real-time mental stress detection based on smartwatch. In: 2017 IEEE International Conference on Consumer Electronics (ICCE), pp. 110-111. IEEE, (2017)

34.Quiroz, J.C., Geangu, E., Yong, M.H.: Emotion Recognition Using Smart Watch Sensor Data: Mixed-Design Study. JMIR mental health 5, e10153 (2018)

35.Zhang, Z., Song, Y., Cui, L., Liu, X., Zhu, T.: Emotion recognition based on customized smart bracelet with built-in accelerometer. PeerJ 4, e2258 (2016)

36.Deterding, S., Dixon, D., Khaled, R., Nacke, L.: From game design elements to gamefulness: defining gamification. In: Proceedings of the 15th international academic MindTrek conference: Envisioning future media environments, pp. 9-15. ACM, (2014)

37.Deterding, S., Sicart, M., Nacke, L., O'Hara, K., Dixon, D.: Gamification. using game-design elements in non-gaming contexts. In: CHI'11 extended abstracts on human factors in computing systems, pp. 2425-2428. ACM, (2011)

38.Stokes, B.: Videogames have changed: time to considerSerious Games'? Development Education Journal 11, 12 (2005)

39.Hamari, J., Koivisto, J., Sarsa, H.: Does gamification work?--a literature review of empirical studies on gamification. In: 2014 47th Hawaii international conference on system sciences (HICSS), pp. 3025-3034. IEEE, (2014)

40.McCallum, S.: Gamification and serious games for personalized health. Stud Health Technol Inform 177, 85-96 (2012)

41.Brauner, P., Valdez, A.C., Schroeder, U., Ziefle, M.: Increase physical fitness and create health awareness through exergames and gamification. Human factors in computing and informatics, pp. 349-362. Springer (2013)

42.Boillat, T., Rivas, H., Wac, K.: "Healthcare on a Wrist": Increasing Compliance Through Checklists on Wearables in Obesity (Self-) Management Programs. Digital Health, pp. 65-81. Springer (2018)

43.Boletsis, C., McCallum, S., Landmark, B.F.: The use of smartwatches for health monitoring in home-based dementia care. In: International Conference on Human Aspects of IT for the Aged Population, pp. 15-26. Springer, (2015)

44.Boletsis, C., McCallum, S.: Connecting the player to the doctor: utilising serious games for cognitive training \& screening. Designing Self-care for Everyday Life. 5 (2014)

45.Gimpel, H., Nißen, M., Görlitz, R.: Quantifying the quantified self: A study on the motivations of patients to track their own health. (2013) 